\title{
Primary Sjogren's Syndrome Presenting as Hypokalaemic Periodic Paralysis- A Rare Case Report
}

\author{
Deep Hathi ${ }^{1}$, Samarth Shukla ${ }^{2}$, Ayush Somani ${ }^{3}$, Anusha Gupta ${ }^{4}$, Sourya Acharya ${ }^{5}$
}

\begin{abstract}
${ }^{1}$ Department of Medicine, Datta Meghe Institute of Medical Sciences (Deemed to be University), Meghe, Wardha, Maharashtra, India. ${ }^{2}$ Department of Pathology, Datta Meghe Institute of Medical Sciences (Deemed to be University), Meghe, Wardha, Maharashtra, India. ${ }^{3}$ Department of Medicine, Datta Meghe Institute of Medical Sciences (Deemed to be University), Meghe, Wardha, Maharashtra, India. ${ }^{4}$ Department of Medicine, Datta Meghe Institute of Medical Sciences (Deemed to be University), Meghe, Wardha, Maharashtra, India. ${ }^{5}$ Department of Medicine, Datta Meghe Institute of Medical Sciences (Deemed to be University), Meghe, Wardha, Maharashtra, India.
\end{abstract}

\section{INTRODUCTION}

Sjogren's syndrome is "an autoimmune disorder which is characterized by lymphocytic infiltration of salivary and lacrimal glands". The commonest presenting symptoms are dryness of eyes, mouth and parotid gland enlargement. SS also has extra glandular manifestation in the form of RTA (renal tubular acidosis) seen in onethird cases. RTA presenting as hypokalaemic periodic paralysis is described in few cases in the literature as a rare presentation. We present a case report of 40 -year-old female who presented with progressive weakness in four limbs. Her serum potassium level was low (1.2 meq/L). After thorough investigations she was diagnosed to have primary SS. She was treated with intravenous potassium chloride which lead to gradual improvement in weakness. She was also started on prednisolone $40 \mathrm{mg} /$ day which was tapered gradually. Thus, it is important to keep possibility of SS in patient with unexplained distal RTA or hypokalaemia. The most common connective tissue disorder to be reported is Sjogren's syndrome (SS).(1) It can be either primary or secondarily associated with other connective disorders like RA (rheumatoid arthritis), SLE (systemic lupus erythematosus).

About half the patients with SS are of primary type

1. SS is characterised by auto- antibodies against lacrimal and salivary glands.(1) The most common presentation is of dryness of eyes and mouth. It is also associated with various extra-glandular manifestations

2. Association of primary SS and hypokalaemic periodic paralysis (HPP) was first described in 1981, however it is infrequently reported.(2) The main renal pathology in primary SS is tubulointerstitial nephritis of which renal tubular acidosis is the main presentation, ${ }^{(3)}$ which is reported in 4.3 to $9 \%$ of SS patients and is common in middle-aged women. $(3,4)$ Hypokalaemic paralysis is the primary symptom in $7 \%$ of patients with SS.(5) Hereby, we present a case of middle age female presenting with HPP as initial presentation of primary SS.
Corresponding Author: Dr. Deep Hathi,

F-9, Raghobaji PG Hostel, DMIMS Campus, Sawangi, Meghe, Wardha-442001, Maharashtra, India. E-mail:deephathi18@gmail.com

DOI: $10.14260 / \mathrm{jemds} / 2020 / 191$

Financial or Other Competing Interests: None.

How to Cite This Article:

Hathi D, Shukla S, Somani A, et al. Primary Sjogren's Syndrome Presenting as Hypokalaemic Periodic Paralysis- A Rare Case Report. J. Evolution Med. Dent. Sci. 2020;9(11):891-893, DOI: $10.14260 /$ jemds/2020/191

Submission 16-11-2019,

Peer Review 18-02-2020,

Acceptance 26-02-2020,

Published 16-03-2020.

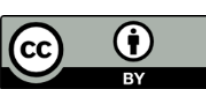




\section{PRESENTATION OF CASE}

A 40-year-old middle aged female presented to emergency department of our hospital with complaints of sudden onset painless weakness of all four limbs, progressive since 1 day. There was no history of sensory loss or autonomic involvement. There was no history of fever, trauma, chronic diarrhoea, palpitations, tremors, excessive sweating and chronic drug use. Also, she denied history of double vision, drooping of eyelid, difficulty in speech or swallowing, sensory complaints or bowel/bladder involvement. No history of similar episodes in past. No $\mathrm{H} / \mathrm{O}$ diabetes mellitus, tuberculosis, hypertension, cardiac, neurological or renal disease. She also gave h/o experiencing dry mouth and eyes since last 3 months.

On examination, patient was conscious oriented, pulse$84 / \mathrm{min}$ regular, $\mathrm{BP}-120 / 80 \mathrm{mmHg}$, respiratory rate- $16 / \mathrm{min}$. CNS examination- hypotonia in all 4 limbs with power being 2/5. Deep tendon reflexes were absent in 4 limbs. Plantar were bilaterally mute and there was no cranial nerve involvement.

\section{PATHOLOGICAL DISCUSSION}

On investigation, she had severe hypokalaemia with s. potassium levels of $1.2 \mathrm{meq} / \mathrm{L}$, normal anion gap (12.5), metabolic acidosis ( $\left.\mathrm{pH}-7.28 \mathrm{HCO}_{3}-12 \mathrm{meq} / \mathrm{L}\right)$ with urinary potassium being $16 \mathrm{meq} / \mathrm{L}$ suggesting renal potassium loss. Serum magnesium was within normal limits. Blood urea (21 $\mathrm{mg} / \mathrm{dl})$ and serum creatinine $(0.9 \mathrm{mg} / \mathrm{dl})$ were within normal range. A diagnosis of distal RTA was kept in view of normal anion gap metabolic acidosis, alkaline urine and hypokalaemia. Thyroid function test was normal. Autoimmune work-up of patients showed ANA positive, anti-dsDNA negative and strongly positive anti-Ro (SS-A) $85.6 \mathrm{U} / \mathrm{ml}(\mathrm{N}<$ 3.0) and anti- LA (SS-B) $80.3(\mathrm{~N}<3.0)$ suggestive of possible primary SS. Lip biopsy was taken which showed lymphocyte infiltrate in minor salivary gland and features suggestive of chronic sialadenitis (figure 1). Schirmer's test was positive. Hence a diagnosis of Sjogren syndrome was made as per SICCA guidelines wherein all the 3 criteria were positive ("serology for Anti-SS-A and SS-B antibody plus salivary gland biopsy and presence of symptoms").

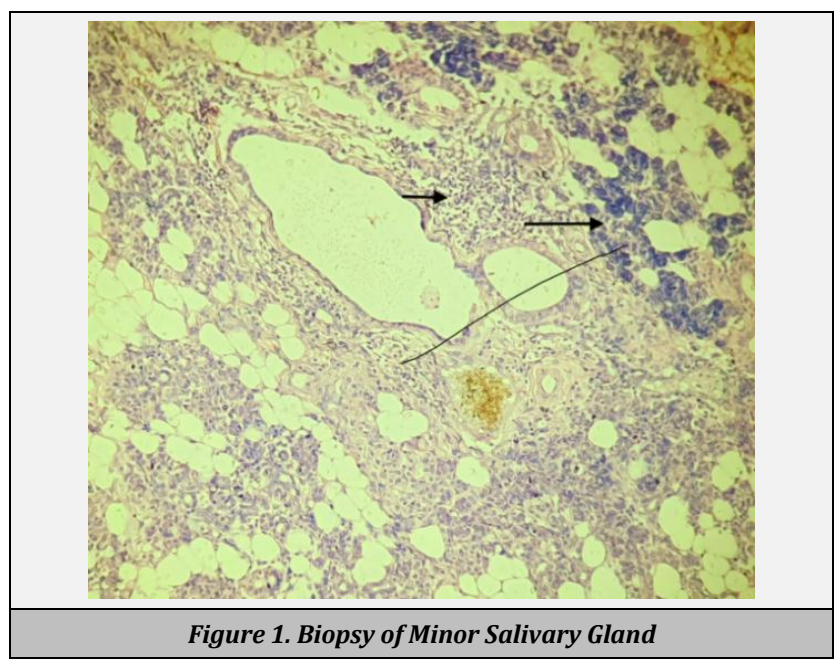

Section from H \& E stained slide 10X view shows acinar, glandular and ductal structures with extensive chronic nonspecific lymphocytic inflammatory infiltrate (short arrow) around the periductal and peri acinar structures at place atrophied acinar structures can be appreciated (long arrow). Histopathological features are suggestive of chronic sialadenitis.

\section{DISCUSSION OF MANAGEMENT}

She was treated with intravenous potassium followed by oral potassium citrate with gradual improvement in weakness over next 2 days with normalisation of potassium levels. In view of systemic involvement of $\mathrm{SS}$, she was started on oral prednisolone $40 \mathrm{mg} /$ day, which was gradually tapered to 10 $\mathrm{mg} /$ day. She was followed up for next 3 months with gradual reduction in requirement of potassium citrate and tapering of prednisolone to $5 \mathrm{mg} /$ day.

\section{DISCUSSION}

Periodic paralysis is a group of disorders characterized by episodes of flaccid paralysis occurring at irregular interval with complete recovery in between attack. Most of the cases are as follows:

1. Hereditary with autosomal dominance inheritance.

2. Associated with alteration of sodium and potassium levels.

3. The various forms being hypokalaemic, hyperkalaemic, thyrotoxic, and paramyotonia congenital.

4. Symptoms are most evident during childhood and adolescence and are triggered by large carbohydrate meal, vigorous exercise, and viral illness.

The patient in our case had severe hypokalaemia with acute flaccid paralysis of all four limbs. However, the age of onset being above 50 years, the likelihood of being an inherited condition becomes a remote possibility. Evaluation and careful investigation of hypokalaemia lead to diagnosis of metabolic acidosis with normal anion gap with urine $\mathrm{pH}$ of $>5$ which in turn revealed distal RTA Type 1 . When the causes of distal RTA were investigated, high-positive Sjogren antibodies were found. Further, examination of eyes, mouth revealed severe dryness with positive Schirmer's test, and lip biopsy showed chronic lymphocytic sialadenitis. Our patient was diagnosed as a case of primary Sjogren syndrome based on diagnostic criteria by Sjogren's international collaborative clinical alliance (SICCA 2012),(6) which included

1. Positive serum anti-SSA (Ro) and/or anti-SSB (La).

2. Labial salivary gland biopsy exhibiting focal lymphocytic sialadenitis with a focus score of $>1$ focus $/ 4 \mathrm{~mm}$.

3. Keratoconjunctivitis sicca with ocular staining score $>3$.

Sjogren syndrome is "chronic systemic autoimmune disorder characterized by lymphocytic infiltration of exocrine glands". The disease can present as alone (primary Sjogren syndrome) or associated with other autoimmune conditions such as SLE, rheumatoid arthritis, and scleroderma (secondary 
Sjogren syndrome). The prevalence of primary Sjogren's syndrome is $\sim 0.5 \%-1 \%$ and middle-aged women (female-to-male ratio is 9:1) are primarily affected.(7) Most patients presents with sicca symptoms such as xerophthalmia, xerostomia, and parotid gland enlargement. In addition, various extra glandular manifestations like arthralgia, arthritis, vasculitis, lymphoma, renal involvement, and Raynaud's phenomenon may be seen.(8) Renal involvement includes proximal and distal tubular acidosis, tubular proteinuria, and nephrogenic diabetes insipidus.(9) The mechanism of hypokalaemia in Sjogren syndrome is because of distal RTA brought about by chronic interstitial nephritis which in turn leads to decrease tubular sodium delivery, defective H-KATPase, secondary hyperaldosteronism, and bicarbonaturia. Thus, it is recommended that all the cases of periodic paralysis should be evaluated for Sjogren's syndrome.

\section{REFERENCES}

[1] Pillemer SR, Matteson EL, Jacobsson LT, et al. Incidence of physician-diagnosed primary Sjögren's syndrome in residents of Olmsted County, Minnesota. Mayo Clin Proc 2001;76(6):593-9.

[2] Raskin RJ, Tesar JT, Lawless OJ. Hypokalaemic periodic paralysis in Sjögren's syndrome. Arch Intern Med 1981;141(12):1671-3.
[3] Ramos-Casals M, Brito-Zeron $P$, Seror R, et al. Characterization of systemic disease in primary Sjogren's syndrome: EULAR-SS Task Force recommendations for articular, cutaneous, pulmonary and renal involvements. Rheumatology (Oxford) 2015;54(12):2230-8.

[4] Ramos-Casals M, Brito-Zeron P, Solans R, et al. Systemic involvement in primary Sjogren's syndrome evaluated by the EULAR-SS disease activity index: Analysis of 921 Spanish patients (GEAS-SS registry). Rheumatology (Oxford) 2014;53(2):321-31.

[5] Ren H, Wang WM, Chen XN, et al. Renal involvement and followup of 130 patients with primary Sjogren's syndrome. The Journal of Rheumatology 2008;35(2):27884.

[6] Shiboski SC, Shiboski CH, Criswell LA, et al. American college of rheumatology classification criteria for Sjögren's syndrome: a data-driven, expert consensus approach in the Sjögren's International Collaborative Clinical Alliance Cohort. Arthritis Care Res (Hoboken) 2012;64(4):475-87.

[7] Fox RI. Sjögren's syndrome. The Lancet 2005;366(9482):321-31.

[8] Bossini N, Savoldi S, Franceschini F, et al. Clinical and morphological features of kidney involvement in primary sjögren's syndrome. Nephrol Dial Transplant 2001;16(12):2328-36.

[9] Maripuri S, Grande JP, Osborn TG, et al. Renal involvement in primary Sjögren's syndrome: a clinicopathologic study. Clin J Am Soc Nephrol 2009;4(9):1423-31. 